\title{
The effectiveness of gabapentin and exercises in the treatment of carpal tunnel syndrome: a randomized clinical trial
}

\author{
Omid Hesami', Mahsa Haghighatzadeh', Behnam Safarpour Lima', Naghmeh Emadi², Shahin Salehi, ${ }^{3 * *}$ \\ 'Department of Neurology, Imam Hossein Hospital, Shahid Beheshti University of Medical Science, Tehran, Iran \\ ${ }^{2}$ Dentofacial Deformities Research Center, Research Institute of Dental Sciences, Shahid Beheshti University of Medical Sciences, Tehran, Iran \\ ${ }^{3}$ Department of Sports Medicine, Imam Hossein Hospital, Shahid Beheshti University of Medical Sciences, Tehran, Iran
}

Carpal tunnel syndrome (CTS) is one of the reasons for labor abandonment due to inability and pain. The aim of this study was to evaluate the effectiveness of gabapentin and exercise training in the treatment of CTS and compare their effects. This single-blind clinical trial was conducted on patients referred to the Imam Hossein hospital's electrodiagnostic (EDX) unit. The patients randomly assigned into four groups: using nocturnal splint as an approved treatment in the control group; taking 300-mg gabapentin per night and using nocturnal splint; nerve and tendon gliding exercises and using nocturnal splint; and taking 300-mg gabapentin per night, performing same exercise as group 3 and using nocturnal splint. At baseline, four indicators were assessed in all patients, including the Boston carpal tunnel questionnaire, visual analogue scale (VAS), pinch and grip strength of the affected hand. One month after the beginning of intervention, participants were reassessed and compared for each of the four indicators. Using nocturnal splint along with exercise and gabapentin significantly improved VAS, pinch and grip strength in moderate CTS compared to control group that only used nocturnal splint. However in mild CTS, grip strength was not significantly higher compared to control group $(P=0.048)$. Results of this study showed that use of splint alone in mild CTS is an appropriate and sufficient treatment; however, in moderate CTS, receiving gabapentin along with exercise and splinting showed better treatment results compared to splinting alone.

Keywords: Boston carpal tunnel questionnaire, Carpal tunnel syndrome, Exercise, Gabapentin, Grip and pinch strength, Visual analogue scale

\section{INTRODUCTION}

Carpal tunnel syndrome (CTS) is a chronic compressive neuropathy that is caused by median nerve entrapment in the carpal tunnel and is the most common entrapment neuropathy (Aroori and Spence, 2008; Park et al., 2017). This syndrome is the most expensive upper extremities musculoskeletal problem. In the United States, it costs about $\$ 10$ billion annually (due to the need for surgery) (Stapleton, 2006).

Carpal tunnel is an osteofibrous canal that contains nine flexor tendons (flexor pollicis longus, four flexor digitorum superficialis, four flexor digitorum profundus) and median nerve (Raja and Carlos, 2015). CTS is manifested by paresthesia (exacerbating at

night), numbness, tingling, and pain in the median nerve area (Bland et al., 2011; Eftekharsadat et al., 2015).The prevalence of the disease is $5.3 \%$ in women and $2.1 \%$ in men (Shiri et al., 2009) and CTS has a prevalence rate of $7.8 \%$ and annual incidence rate of $5.7 \%$ in the working population (Dale et al., 2013).

Risk factors reported for CTS include diabetes (Pourmemari and Shiri, 2016), hypothyroidism (Karne and Bhalerao, 2016), rheumatologic diseases and occupational factors (Petit et al., 2015). In addition to the cost of surgical treatment, there are various reasons for the choice of nonsurgical and preservative treatment, including postoperative complications such as infection, nerve damage, tendon injury, and reflex sympathetic dystrophy syndrome (Osterman et al., 2002). Therefore, the selection of appropriate nonsur-
${ }^{\star}$ Corresponding author: Shahin Salehi (D) https://orcid.org/0000-0002-2874-0436 Department of Sports Medicine, Imam Hossein hospital, Shahid Beheshti University of Medical Science, Tehran, Iran

Tel: +98-9121406684, Fax:+98-2122427753, E-mail: Salehi2955@yahoo.com Received: August 19, 2018 / Accepted: November 2, 2018
This is an Open Access article distributed under the terms of the Creative Commons Attribution Non-Commercial License (http://creativecommons.org/licenses/by-nc/4.0/) which permits unrestricted non-commercial use, distribution, and reproduction in any medium, provided the original work is properly cited. 
gical treatment is very important when facing this common and costly disease.

Gabapentin is one of the medications used in neuropathic pain which is effective in reducing neuropathic pain in trigeminal neuralgia, diabetic neuropathy, post herpetic neuralgia, and reflex sympathetic dystrophy syndrome (Backonja et al., 1998; Rowbotham et al., 1998; Rullán et al., 2017). There are some discrepancies in literature about the degrees of effectiveness of gabapentin (Eftekharsadat et al., 2015; Hui et al., 2011) and exercises in treatment of CTS (Bialosky et al., 2009; Tal-Akabi and Rushton, 2000).

In the severe type and advanced stages of the CTS, the median nerve gets an hour-glass deformity due to narrowing under the thickened flexor retinaculum with swelling over the distal and proximal region, and the nerve injury is irreversible (Nakamichi and Tachibana, 2000). Therefore, the study of noninvasive treatments in early stages of the disease is necessary in order to prevent the progression of the disease, waste of time, cost; and to obtain the most comprehensive treatment. The aim of this study was to evaluate the effectiveness of splinting, gabapentin, exercises and combinations of these conservative therapies in treatment of mild to moderate CTS.

\section{MATERIALS AND METHODS}

\section{Trial design}

This study was approved by the Ethics Committee of the Shahid Beheshti University of Medical Sciences in Iran (IR.SBMU. MSP.REC.A396.59). The study was a single-blind clinical controlled trial that was conducted in patients referred to the electrodiagnostic (EDX) unit of Imam Hossein hospital.

\section{Inclusion criteria}

The inclusion criteria of this study were: (a) presence of sensory symptoms in median nerve area for more than 3 months, and (b) EDX evidence of entrapment of median nerve due to CTS. People who met one of the following criteria were excluded from the study: evidence of severe entrapment including atrophy of the thenar muscles and EDX studies indicating severe nerve entrapment (due to the need for referral for surgery); EDX and clinical examination evidence of conditions that mimic the CTS such as cervical radiculopathy, polyneuropathy, and proximal median neuropathies; taking antiepileptic and tricyclic antidepressant for other reasons; taking oral and injectable corticosteroids in the last three months; diabetes mellitus; hypothyroidism; pregnancy; rheumatologic diseases; trauma; doing similar exercises during last month; history of suicidal ideation or suicidal attempts; and a creatinine clearance level less than 30. People who were sensitive to side effects of gabapentin or those who were not able to continue their participation in the study were excluded and replaced.

\section{Intervention}

Patients with mild to moderate CTS were randomly assigned to one of the four intervention groups:

(a) Using nocturnal cock-up splint as an approved treatment in the control group.

(b) Taking 300-mg gabapentin per night and using nocturnal splint.

(c) Using nocturnal splint and performing daily exercises that each type of exercise should be done 10 times per day. The exercises include nerve gliding exercise, tendon gliding exercise, stretching, and griping.

(d) Taking 300-mg gabapentin per night, performing same exercise as group 3 and using nocturnal splint.

The diagnosis of CTS was based on the presence of paresthesia and pain with the same characteristics of CTS' symptoms. The diagnosis was confirmed and severity of CTS was determined using electromyography (EMG). The following definition for severity of CTS (mild, moderate, and severe) based on EMG evidences was used in this study:

Mild: median distal sensory peak latency prolongation, either absolute or relative (using sensitive comparative methods) and no evidence of axon loss.

Moderate: abnormal median sensory latency, as noted in the mild type, and absolute or relative prolongation of median motor distal latency (using sensitive comparative methods), and lack of evidence of axon loss.

Severe: any disturbances in nerve conduction studies mentioned above, along with evidence of axon loss, defined as: (a) lack or decreased amplitude of sensory nerve action potential or mixed nerve action potential of median nerve (b) lack of or decreased amplitude of thenar compound muscle action potential; or (c) the presence of fibrillation potentials or changes in motor unit potentials such as increasing amplitude, duration or phases in needle EMG (Werner and Andary, 2011).

\section{Randomization}

All patients gave informed consent before being entered into the study. Side effects of the medication were explained to patients. Participants were advised to stop taking the medication if they have side effects. Recruitment to the trial was done using 
convenience sampling. The randomization of patients for assigning to intervention groups was done through stratified permutation blocks method, where the severity of the disease was taken into account as a block.

\section{Measurement}

The following measures were used to assess patients before and 1 month after treatment: (a) Visual analogue scale (VAS) for assessment of pain and paresthesia; (b) Boston carpal tunnel questionnaire (BCTQ) including symptom severity scale (SSS) and functional status scale (FSS) for evaluating the treatment effectiveness; (c) Grip strength was measured by SAEHAN hydraulic handgrip dynamometer (Model SH5001; SAEHAN Corp., Seoul, Korea); (d) Pinch strength was measured by SAEHAN hydraulic pinch dynamometer (Model SH5005).

\section{Self-report questionnaire}

Severity of symptoms and functional status were assessed using the BCTQ that is a self-administered and self-report questionnaire (Levine et al., 1993). The BCTQ consists of two parts: SSS and FSS. The SSS evaluates symptoms such as pain, numbness, weakness, and paresthesia using 11 questions. FSS evaluates problems in everyday activities such as writing, dressing, keeping a book while reading, and etc. using eight questions. The SSS items' score can range from 1 (no symptoms) to 5 (severe). The FSS items' score can range from 1 (no dysfunction) to 5 (disability due to symptoms).

Pain and paresthesia were measured using VAS. The severity of pain was categorized between 0 and 10 , in which 0 means no pain and 10 means the worst possible pain. Patients were asked to match their pain according to VAS scale (Eftekharsadat et al., 2015).

\section{Performance-based outcome measures}

To measure grip strength, a dynamometer was used which was a sensitive and repeatable tool. First, the participants were taught how to use the device. Then, patients were asked to do the test once to familiarize with the device. After giving rest to their wrist, the actual test was performed. Participants were given three chances to apply their maximum force, and the average was calculated.

A pinch meter was used to measure pinch strength and maximum force in palmar pinch was measured.

\section{Tendon gliding exercises}

All patients were trained by a sports medicine specialist on ten- don and nerve gliding exercises that patients should do at home. In these exercises, fingers are located in five different positions including straight, hook, fist, tabletop, and straight fist.

\section{Nerve gliding exercise}

In order to enable the mobilization of the median nerve, patients need to perform the exercise in a way that the hand and wrist would be in six different positions:

(a) The wrist is in neutral position, the fingers and thumb are in flexion.

(b) The wrist is in neutral position, the fingers and thumb are in extension.

(c) The wrist and fingers are in extension, the thumb is in neutral position.

(d) The wrist, fingers, and thumb are in extension.

(e) As position 4, but forearm is in supination.

(f) As position 5, but gently stretching the thumb with the other hand (Madenci et al., 2012).

Two hundred thirty patients were referred to the sports medicine clinic of Imam Hossein hospital after their diagnosis was confirmed by EDX studies. Preintervention assessments were done by a neurologist and provided to the sports medicine specialist. The sports medicine specialist identified four treatment groups based on the permutation blocks randomization method (was done by statistician), while the neurologist did not know about the type of treatment group. The sample size was calculated considering the attrition rate. Therapeutic exercises were taught to the patients in groups 3 and 4 by a sports medicine specialist, and a sheet with of photos of each step was provided to these groups. After 1 month, patients were reassessed by the neurologist using BCTQ, VAS, pinch and grip strength with a dynamometer, while the neurologist was not aware of the patient's treatment group. The data were entered in the table by the sports medicine specialist.

\section{Analysis}

Distribution of underlying variables such as gender, body mass index (BMI), disease state, and age in intervention groups, was analyzed using one-way analysis of variance (ANOVA), chi-square and Fisher exact test, depending on the type of variables. Finally, one-way ANOVA was used to evaluate the effect of intervention groups on the difference between postintervention scores and baseline scores. $P$-value less than 0.05 was considered meaningful. All analyzes were performed using IBM SPSS Statistics ver. 22.0 (IBM Co., Armonk, NY, USA). Tukey post boc test was used to 


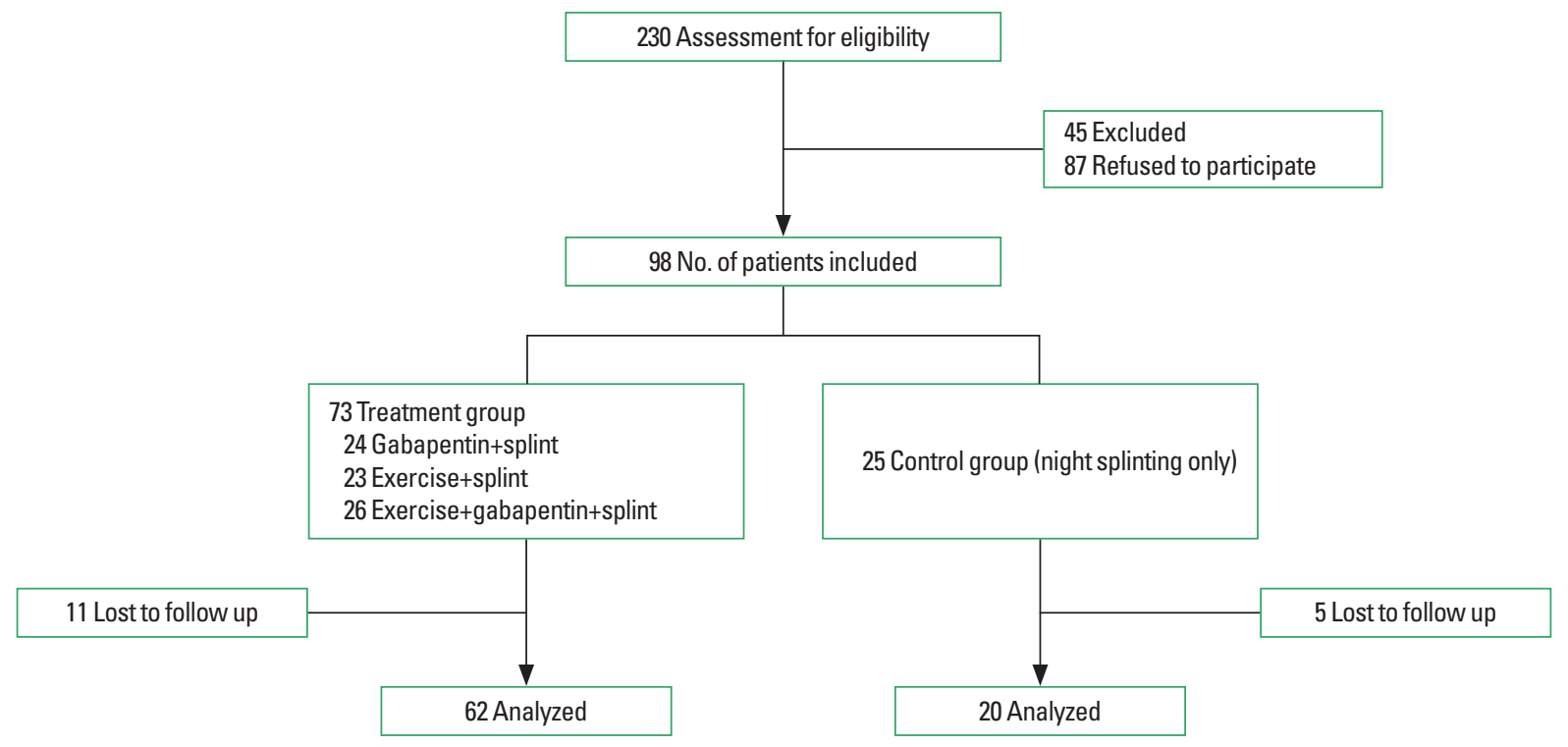

Fig. 1. Flow chart of trial and number of patients. From 230 patients of eligibility, 98 patients were randomized and 82 patients completed the course of intervention.

compare each group with other groups. Dunnett post hoc test was used to compare each group with the control group. Analysis of covariance was used to compare postintervention response values among the intervention groups. In this method, the dependent variable at baseline, along with other underlying variables as covariates, was entered into the model, so that its effect, if available, can be considered on the response.

\section{RESULTS}

A total of 82 out of 230 patients (eligible) were entered in the study and completed the 1-month follow-up (Fig. 1). In this study, $88 \%$ of participants were female. Most of the patients were overweight or obese. BMI of about $88 \%$ of patients was greater than normal. Majority of participants (67.1\%) has mild CTS.

As presented in Table 1, distribution of BMI and gender are not the same among groups, while the severity of the disease is roughly distributed the same among groups. There was a statistically significant difference between the groups in terms of mean age of the patients (Table 1). The youngest patient was 24 years old and the oldest patient was 74 years old. Half of all patients were over 46 years of age.

As shown in Table 2, in patients with mild CTS, there was no statistically significant difference between groups in postintervention mean of Boston.SSS, Boston.FSS, pinch strength, and VAS, indicating no difference between intervention groups and control group. However, grip strength was not significantly higher in
Table 1. Demographic data and preintervention status of patients in different groups

\begin{tabular}{lccccr}
\hline Characteristic & Exercise & Gabapentin & $\begin{array}{c}\text { Exercise+ } \\
\text { gabapentin }\end{array}$ & Control & $P$-value \\
\hline Age (yr) & $54.17 \pm 9.99$ & $41.04 \pm 8.46$ & $47.13 \pm 9.14$ & $48.30 \pm 9.96$ & $<0.001$ \\
Gender & & & & 0.006 \\
Male & $6(26.1)$ & $0(0)$ & $0(0)$ & $4(20)$ & \\
Female & $17(73.9)$ & $23(100)$ & $16(100)$ & $16(80)$ & \\
BMla) & & & & & 0.04 \\
Normal & $2(8.7)$ & $0(0)$ & $5(31.3)$ & $3(15)$ & \\
Overweight & $9(39.1)$ & $12(52.2)$ & $9(56.3)$ & $9(45)$ & \\
Obesity grade I & $7(30.4)$ & $10(43.5)$ & $2(12.5)$ & $7(35)$ & \\
Obesitygrade II & $5(21.7)$ & $1(4.3)$ & $0(0)$ & $1(5)$ & \\
Severity & & & & & 0.246 \\
Mild & $13(56.5)$ & $19(82.6)$ & $11(68.8)$ & $12(60)$ & 0.035 \\
Moderate & $10(43.5)$ & $4(17.4)$ & $5(31.3)$ & $8(40)$ & 0.068 \\
Boston.SSS & $20.52 \pm 10.05$ & $22.43 \pm 7.75$ & $27.31 \pm 7.60$ & $25.60 \pm 4.25$ & $<0.001$ \\
Boston.FSS & $21.52 \pm 10.56$ & $18.30 \pm 9.49$ & $25.38 \pm 8.34$ & $19.20 \pm 3.43$ & $<0.001$ \\
Grip strength & $23.28 \pm 7.60$ & $20.04 \pm 6.91$ & $14.06 \pm 6.45$ & $15.65 \pm 4.78$ & 0.422 \\
Pinch strength & $6.76 \pm 1.48$ & $5.80 \pm 2.09$ & $3.75 \pm 1.92$ & $5.85 \pm 1.51$ & $<0.001$ \\
VAS & $6.30 \pm 2.58$ & $6.74 \pm 2.78$ & $7.19 \pm 2.14$ & $6.00 \pm 0.97$ & 0.422 \\
\hline
\end{tabular}

Values are presented as mean \pm standard deviation or number (\%).

BMI, body mass index; Boston, Boston carpal tunnel questionnaire; SSS, symptom severity scale; FSS, functional status scale; VAS, visual analogue scale.

alBMI (kg/m²): normal, 18.5-24.9; overweight, 25.0-29.9; obesity grade I, 30.0-34.9; obesity grade II, 35.0-39.9.

group 4 (gabapentin+exercise) compared to control group $(P=$ $0.048)$.

In patients with moderate CTS, there was no significant differ- 
Table 2. Postintervention mean for mild and moderate group and the whole sample

\begin{tabular}{|c|c|c|c|c|c|c|}
\hline \multirow{2}{*}{ Outcome } & \multicolumn{2}{|c|}{ Mild* } & \multicolumn{2}{|c|}{ Moderate* $^{*}$} & \multicolumn{2}{|c|}{ Total $^{\dagger}$} \\
\hline & Mean \pm SD & $P$-value & Mean \pm SD & $P$-value & Mean \pm SD & $P$-value \\
\hline Boston.SSS & & 0.26 & & 0.449 & & 0.104 \\
\hline Exercise+gabapentin & $19.91 \pm 8.5$ & & $15.40 \pm 7.99$ & & $18.50 \pm 8.36$ & \\
\hline Exercise & $15.31 \pm 9.48$ & & $18.90 \pm 10.46$ & & $16.87 \pm 9.85$ & \\
\hline Gabapentin & $20.42 \pm 5.33$ & & $16.00 \pm 3.65$ & & $19.65 \pm 5.29$ & \\
\hline Control & $20.92 \pm 3.15$ & & $21.88 \pm 5.25$ & & $21.30 \pm 4.01$ & \\
\hline Boston.FSS & & 0.701 & & 0.023 & & 0.895 \\
\hline Exercise+gabapentin & $24.45 \pm 6.23$ & & $17.20 \pm 7.16^{\mathrm{al}}$ & & $22.19 \pm 7.19$ & \\
\hline Exercise & $16.85 \pm 11.55$ & & $21.90 \pm 10.00^{b)}$ & & $19.04 \pm 10.97$ & \\
\hline Gabapentin & $17.84 \pm 8.98$ & & $16.75 \pm 7.18$ & & $17.65 \pm 8.56$ & \\
\hline Control & $16.75 \pm 3.55$ & & $17.25 \pm 2.71$ & & $16.95 \pm 3.17$ & \\
\hline Grip strength & & 0.048 & & 0.021 & & 0.023 \\
\hline Exercise+gabapentin & $19.86 \pm 8.35^{a)}$ & & $16.40 \pm 9.40^{\mathrm{al}}$ & & $18.78 \pm 8.53^{\mathrm{a})}$ & \\
\hline Exercise & $25.69 \pm 4.03$ & & $22.75 \pm 7.80$ & & $24.41 \pm 6.00$ & \\
\hline Gabapentin & $20.16 \pm 5.89$ & & $22.50 \pm 1.73$ & & $20.57 \pm 5.44$ & \\
\hline Control & $16.08 \pm 3.87^{b)}$ & & $18.38 \pm 6.35^{b)}$ & & $17.00 \pm 4.98$ & \\
\hline Pinch strength & & 0.619 & & 0.001 & & 0.19 \\
\hline Exercise+gabapentin & $5.64 \pm 2.09$ & & $6.10 \pm 2.38^{a)}$ & & $5.78 \pm 2.11$ & \\
\hline Exercise & $7.42 \pm 1.22$ & & $7.10 \pm 1.93^{b)}$ & & $7.28 \pm 1.54$ & \\
\hline Gabapentin & $6.40 \pm 1.62$ & & $7.75 \pm 1.26^{b l}$ & & $6.63 \pm 1.63$ & \\
\hline Control & $6.17 \pm 1.15$ & & $7.13 \pm 2.23^{b l}$ & & $6.55 \pm 1.69$ & \\
\hline VAS & & 0.778 & & 0.015 & & 0.187 \\
\hline Exercise+gabapentin & $4.30 \pm 2.06$ & & $4.20 \pm 2.28^{a l}$ & & $4.27 \pm 2.05$ & \\
\hline Exercise & $4.15 \pm 2.23$ & & $4.70 \pm 2.63^{b)}$ & & $4.39 \pm 2.37$ & \\
\hline Gabapentin & $5.11 \pm 2.18$ & & $2.00 \pm 2.83$ & & $4.57 \pm 2.54$ & \\
\hline Control & $4.75 \pm 1.06$ & & $5.00 \pm 1.07^{b l}$ & & $4.85 \pm 1.04$ & \\
\hline
\end{tabular}

SD, standard deviation; Boston, Boston carpal tunnel questionnaire; SSS, symptom severity scale; FSS, functional status scale; VAS, visual analogue scale.

${ }^{*}$ Analysis of covariance - adjusted for baseline measurements, age, gender, body mass index (BMI). ${ }^{\dagger}$ Analysis of covariance - adjusted for baseline measurements, age, gender, BMl, severity of the disease. Different letters ${ }^{\left({ }^{a, b}\right)}$ indicate significant differences (group marked by letter ${ }^{\mathrm{a} /}$ shows significantly better performance).

ence in BCTQ indices after treatment comparing with control group; but in pinch, grip, and VAS, there was a significant difference in mean after treatment. This difference was observed in those who received gabapentin and did exercises.

\section{DISCUSSION}

CTS treatments are based on the removal of pressure from median nerve and according to literature, using splint is an appropriate treatment for mild and moderate CTS (Baysal et al., 2006; Premoselli et al., 2006). Nocturnal splints usually are prescribed for patient in order to prevent interference with daily activities. Another method of reducing the pressure of the carpal tunnel is to stop the inflammation using anti-inflammatory agents (Eftekharsadat et al., 2011). A recent systematic review study reported that according to experimental evidence, oral steroids, and cor- ticosteroid injections help patients with CTS in the short term, however, they are not effective in long term (Huisstede et al., 2018). Given systemic complications of oral steroids and potential side effects of diuretics on cardiovascular system, some studies reported using other drug combinations for treatment of CTS (Eftekharsadat et al., 2011). Because gabapentin is used in treatment of neuropathic pain, in some studies it has been used to treat CTS, including a double-blind clinical trial conducted in 2010 in which 140 patients recently diagnosed with CTS were assigned to two groups receiving either placebo or 900-mg gabapentin daily for at least 3 months. The global symptom score (GSS) was used at baseline, 2 and 8 weeks. GSS score is based on how patients rate their symptoms of pain, numbness, tingling, weakness and clumsiness, and nightly awakening. According to the results, there was no statistically significant difference between the group receiving gabapentin and the group that received placebo (Hui et al., 2011). 
In contrast to the above mentioned study, in another study, patients with mild to moderate CTS (diagnosed based on clinical signs and EDX evidence) were assigned into three treatment groups: (a) control group, (b) 100-mg gabapentin daily, and (c) 300-mg gabapentin daily. All three groups received splint. Patients were assessed at baseline and after 2 months in terms of five indicators including pinch and grip strength, VAS, BCTQ, and NCS. The two treatment groups got better scores comparing to control group for all measures except for NCS. The participants who received 300-mg gabapentin had significantly better scores in SSS and pinch strength compared with those who received 100-mg gabapentin (Eftekharsadat et al., 2015).

According to our study, there was no significant difference between patients who received gabapentin and used splint and control group in patient with mild and moderate CTS. However, there was a significant difference between group 4 (gabapentin + splint + exercise) and control group, especially in the moderate CTS.

In another study conducted in 2018, 41 patients assigned to two groups, one received splints and the other one received splint, tendon/nerve gliding exercise and ultrasound for treatment of CTS. They were evaluated pre and postintervention using BCTQ and no significant difference between the two groups was reported. In this study, drug combinations such as gabapentin have not been used and therapeutic outcomes have been reported in one general group with patients with different severities, and have not been studied in the mild to moderate severity group (Sim et al., 2018).

In another study, manipulative method that included griping and stretching movements was compared with the control group (without treatment). Participants were assessed using daily VAS, the functional table completed by participants and range of motion test. Patients in manipulative method group showed significant improvement compared to control group. In this study, the severity of CTS was not determined and it was only stated that more patients in control group were in need of surgery compared to intervention group (Tal-Akabi and Rushton, 2000).

To compare the effect of neutral wrist and metacarpophalangeal (MCP) splint with a wrist cock-up splint with and without exercise, 61 patients with mild to moderate CTS were assigned to four groups; of those, 51 patients completed the treatment. Participants were assessed using the Boston SSS and FSS at baseline, 4 weeks and 8 weeks. The results showed that participants who used neural wrist and MCP splint had better outcomes compared to those who used a cock-up splint. Exercise was not significantly effective in comparison to the splint group (Brininger et al., 2007).
In a systematic review study that focused on the therapeutic role of nerve gliding exercises in treating patients with CTS, it has been reported that a few studies have found nerve gliding exercises an effective method in treatment of patients with CTS. Authors of this study emphasized on the need for more studies to find out the effectiveness of available treatments for CTS (Ballestero-Pérez et al., 2017).

This study showed that using nocturnal splint along with exercise and taking gabapentin improved VAS, pinch and grip strength compared to control group that only used the nocturnal splint, especially in moderate CTS.

\section{CONFLICT OF INTEREST}

No potential conflict of interest relevant to this article was reported.

\section{REFERENCES}

Aroori S, Spence RA. Carpal tunnel syndrome. Ulster Med J 2008;77:6-17. Backonja M, Beydoun A, Edwards KR, Schwartz SL, Fonseca V, Hes M, LaMoreaux L, Garofalo E. Gabapentin for the symptomatic treatment of painful neuropathy in patients with diabetes mellitus: a randomized controlled trial. JAMA 1998;280:1831-1836.

Ballestero-Pérez R, Plaza-Manzano G, Urraca-Gesto A, Romo-Romo F, Atín-Arratibel ML, Pecos-Martín D, Gallego-Izquierdo T, Romero-Franco N. Effectiveness of nerve gliding exercises on carpal tunnel syndrome: a systematic review. J Manipulative Physiol Ther 2017;40:50-59.

Baysal O, Altay Z, Ozcan C, Ertem K, Yologlu S, Kayhan A. Comparison of three conservative treatment protocols in carpal tunnel syndrome. Int J Clin Pract 2006;60:820-828.

Bialosky JE, Bishop MD, Price DD, Robinson ME, Vincent KR, George SZ. A randomized sham-controlled trial of a neurodynamic technique in the treatment of carpal tunnel syndrome. J Orthop Sports Phys Ther 2009;39:709-723.

Bland JD, Weller P, Rudolfer S. Questionnaire tools for the diagnosis of carpal tunnel syndrome from the patient history. Muscle Nerve 2011;44:757-762.

Brininger TL, Rogers JC, Holm MB, Baker NA, Li ZM, Goitz RJ. Efficacy of a fabricated customized splint and tendon and nerve gliding exercises for the treatment of carpal tunnel syndrome: a randomized controlled trial. Arch Phys Med Rehabil 2007;88:1429-1435.

Dale AM, Harris-Adamson C, Rempel D, Gerr F, Hegmann K, Silverstein B, Burt S, Garg A, Kapellusch J, Merlino L, Thiese MS, Eisen EA, Evanoff $B$. Prevalence and incidence of carpal tunnel syndrome in US 
working populations: pooled analysis of six prospective studies. Scand J Work Environ Health 2013;39:495-505.

Eftekharsadat B, Babaei-Ghazani A, Habibzadeh A. The efficacy of 100 and $300 \mathrm{mg}$ gabapentin in the treatment of carpal tunnel syndrome. Iran J Pharm Res 2015;14:1275-1280.

Eftekharsadat B, Kazem Shakouri S, Shimia M, Rahbar M, Ghojazadeh M, Reza Rashidi M, Hadi Faraji M. Effect of E. laciniata (L) ointment on mild and moderate carpal tunnel syndrome: a double-blind, randomized clinical trial. Phytother Res 2011;25:290-295.

Hui AC, Wong SM, Leung HW, Man BL, Yu E, Wong LK. Gabapentin for the treatment of carpal tunnel syndrome: a randomized controlled trial. Eur J Neurol 2011;18:726-730.

Huisstede BM, Randsdorp MS, van den Brink J, Franke TPC, Koes BW, Hoogvliet P. Effectiveness of oral pain medication and corticosteroid injections for carpal tunnel syndrome: a systematic review. Arch Phys Med Rehabil 2018;99:1609-1622.e10.

Karne SS, Bhalerao NS. Carpal tunnel syndrome in hypothyroidism. J Clin Diagn Res 2016;10:OC36-38.

Levine DW, Simmons BP, Koris MJ, Daltroy LH, Hohl GG, Fossel AH, Katz JN. A self-administered questionnaire for the assessment of severity of symptoms and functional status in carpal tunnel syndrome. J Bone Joint Surg Am 1993;75:1585-1592.

Madenci E, Altindag O, Koca I, Yilmaz M, Gur A. Reliability and efficacy of the new massage technique on the treatment in the patients with carpal tunnel syndrome. Rheumatol Int 2012;32:3171-3179.

Nakamichi KI, Tachibana S. Enlarged median nerve in idiopathic carpal tunnel syndrome. Muscle Nerve 2000;23:1713-1718.

Osterman AL, Whitman M, Porta LD. Nonoperative carpal tunnel syndrome treatment. Hand Clin 2002;18:279-289.

Park YD, Park YJ, Park SS, Lee HL, Moon HH, Kim MK. Effects of taping therapy for carpal space expansion on electrophysiological change in patients with carpal tunnel syndrome. J Exerc Rehabil 2017;13:359362.

Petit A, Ha C, Bodin J, Rigouin P, Descatha A, Brunet R, Goldberg M,
Roquelaure Y. Risk factors for carpal tunnel syndrome related to the work organization: a prospective surveillance study in a large working population. Appl Ergon 2015;47:1-10.

Pourmemari MH, Shiri R. Diabetes as a risk factor for carpal tunnel syndrome: a systematic review and meta-analysis. Diabet Med 2016;33: 10-16.

Premoselli S, Sioli P, Grossi A, Cerri C. Neutral wrist splinting in carpal tunnel syndrome: a 3- and 6-months clinical and neurophysiologic follow-up evaluation of night-only splint therapy. Eura Medicophys 2006;42:121-126.

Raja S, Carlos AJ. Carpal tunnel syndrome - current controversies. Orthop Trauma 2015;29:273-277.

Rowbotham M, Harden N, Stacey B, Bernstein P, Magnus-Miller L. Gabapentin for the treatment of postherpetic neuralgia: a randomized controlled trial. JAMA 1998;280:1837-1842.

Rullán M, Bulilete O, Leiva A, Soler A, Roca A, González-Bals MJ, Lorente P, Llobera J; PHN group. Efficacy of gabapentin for prevention of postherpetic neuralgia: study protocol for a randomized controlled clinical trial. Trials 2017;18:24.

Shiri R, Miranda H, Heliövaara M, Viikari-Juntura E. Physical work load factors and carpal tunnel syndrome: a population-based study. Occup Environ Med 2009;66:368-373.

Sim SE, Gunasagaran J, Goh KJ, Ahmad TS. Short-term clinical outcome of orthosis alone vs combination of orthosis, nerve, and tendon gliding exercises and ultrasound therapy for treatment of carpal tunnel syndrome. J Hand Ther 2018 Feb 7 [Epub]. pii: S0894-1130(17)30221-1. https://doi.org/10.1016/j.jht.2018.01.004.

Stapleton MJ. Occupation and carpal tunnel syndrome. ANZ J Surg 2006; 76:494-496.

Tal-Akabi A, Rushton A. An investigation to compare the effectiveness of carpal bone mobilisation and neurodynamic mobilisation as methods of treatment for carpal tunnel syndrome. Man Ther 2000;5:214-222.

Werner RA, Andary M. Electrodiagnostic evaluation of carpal tunnel syndrome. Muscle Nerve 2011;44:597-607. 